\title{
CO-IMMUNE RETRACEABLE SETS
}

\author{
BY T. G. MCLAUGHLIN
}

Communicated by L. Henkin, December 18, 1964

Our notation and terminology basically follows that found in [2], except with regard to notation for unions and intersections; in a few instances we cite other references for special terms.

The following two propositions are established by a fairly straightforward moveable-markers technique; either proof is only a minor variation on the other. ${ }^{1}$

Proposition A. Let $\alpha$ be an infinite recursively enumerable set. Then there is a countably infinite collection $\Gamma$ of retraceable sets $\gamma_{i}$ such that (i) $i \neq j \Rightarrow \gamma_{i} \cap \gamma_{j}=\varnothing$, (ii) each $\gamma_{i}$ is the unique infinite set retraced by $a$ certain basic general recursive retracing function (for the notions of retracing function and basic retracing function, see [5]), (iii) $\alpha=\mathrm{U} \Gamma$, and (iv) $\alpha-\gamma_{i}$ is immune for all $i$.

Proposition B. Let $\alpha$ be an infinite recursively enumerable set, and $\tau$ an infinite recursive subset of $\alpha$ such that $\alpha-\tau$ is also infinite. Then there is a recursive function $f$ such that, for each $i, f(i)$ indexes a basic, general recursive retracing function which retraces a unique infinite set $\gamma_{i}$, where (i) $i \neq j \Rightarrow \gamma_{i} \cap \gamma_{j}=\varnothing$, (ii) each $\gamma_{i}$ has exactly one number in common with $\alpha^{\prime} \cup \tau$, and (iii) $(\alpha-\tau)-\gamma_{i}$ is immune for all $i$.

It was shown by Yates, in [5] (in answer to a question of Dekker and Myhill), that there are basic retracing functions, some of them retracing unique infinite sets, which do not retrace any infinite recursive set. In each of Yates' examples, all of the sets retraced by such functions have nonimmune complements. The above propositions demonstrate the existence of examples in which an infinite set $\alpha$ is retraced by a basic function and $\alpha$ has immune complement. In any example of this latter type, the function in question must retrace a unique infinite set, which, of course, cannot be recursive.

We remark that all of the sets $\gamma_{i}$ obtained by us in proving Propositions $\mathrm{A}$ and $\mathrm{B}$ are, owing to the nature of the proofs, hyperimmune (for the notion of hyperimmunity, see, e.g., [5]). This is closely related to the following general assertion:

1 We are indebted to Paul Young for a conversation which took place in August, 1963. At that time he made a suggestion which has proved to be susceptible of elaboration into proofs of Propositions A and B. 
Proposition C. Let $\gamma$ be a co-immune retraceable set (i.e., a retraceable set with an immune complement). If $\gamma$ is not hyperimmune, then (a) no regressing function of $\gamma$ is potentially recursive (i.e., has a general recursive extension), and (b) if $p$ is a regressing function for $\gamma$ such that $\rho p \subset \delta p$ (see [2]), then $\delta p$ is not hypersimple. (For the notion of regressing function of a set, see [2]).

It would be of interest to obtain a co-immune, nonhyperimmune retraceable set; for this would yield an example of an immune regressive set all of whose regressing functions fail to be potentially recursive. (Examples of retraceable sets with no potentially recursive retracing functions, and of nonimmune regressive sets with no potentially recursive regressing functions, are known; see [1] and [4].)

We wish to state a corollary to Propositions A and B which involves the notion of cohesive set; some additional terminology is useful in rendering the statement uncluttered. An infinite number set $\alpha$ is called cohesive [4] in case there is no recursively enumerable set $\beta$ for which $\beta \cap \alpha, \beta^{\prime} \cap \alpha$ are both infinite; $\alpha$ is called supercohesive in case this holds relative to regressive sets $\beta$. As was pointed out in [3], every infinite set of numbers has a supercohesive subset, while not all cohesive sets are supercohesive. Let us say that a cohesive set $\alpha$ is of class $\tau$, where $1 \leqq \tau \leqq \aleph_{0}$, in case there is a collection of $\tau$ pairwise disjoint retraceable sets each of which has infinite intersection with $\alpha$ and whose union covers $\alpha$, but there is no such collection of more than $\tau$ retraceable sets.

Proposition D. For every $\tau, 1 \leqq \tau \leqq \aleph_{0}$, there exists a cohesive set of class $\tau$. (In the case $\tau=\aleph_{0}$, it follows from use of Proposition B that the decomposing family of retraceable sets can even be effectively indexed in a certain sense.)

We conclude with a brief discussion of the proofs of Propositions $\mathrm{A}$ and $\mathrm{B}$. No attempt is made to define directly the sets $\gamma_{i}$; rather, a construction is specified for recursive functions which will retrace sets of the required sort. We must make sure that $\gamma_{i}$ is co-immune in $\alpha$ (or $\alpha-\tau$ ); hence, the basic idea of the construction is to tag domain elements of the functions under construction with markers $\Lambda_{i j}$ which we proceed (subject to various constraints) to drive downward, as far as possible, through indices of recursively enumerable subsets of $\alpha$ (or $\alpha-\tau$ ), with the markers which are attached at a given stage being attached to numbers which have been generated (by the stage in question) in recursively enumerable subsets of $\alpha$ (or $\alpha-\tau$ ) given by particular indices under scrutiny at that stage. The upshot of the 
construction is that all markers eventually stop, and $\gamma_{i}$ is the set of numbers $k$ such that the $i$ th constructed function leads down to $k$ from some number $m$ to which a marker $\Lambda_{i j}$ is attached as its final position; for fixed $i, \gamma_{i}$ contains the final positions of all markers $\Lambda_{i j}$, $j>0$, and any infinite recursively enumerable subset of $\alpha$ (or $\alpha-\tau$ ) contains the final position of a marker $\Lambda_{i j}$ for some $j$. Making the various retracing functions total complicates the basic procedure only slightly.

Added in proof. As to the remark following Proposition C: we have since noticed how, by combining a theorem of D. A. Martin with a result in [4], to prove that, indeed, there exists a retraceable set with no potentially recursive regressing function.

\section{REFERENCES}

1. K. I. Appel and T. G. McLaughlin, On properties of regressive sets, Trans. Amer. Math. Soc. 115 (1965), 83-93.

2. J. C. E. Dekker, Infinite series of isols, Proc. Sympos. Pure Math. Vol. 5, pp. 77-96, Amer. Math. Soc., Providence, R. I., 1962.

3. T. G. McLaughlin, Regressive sets, supercohesion, and permutations, Abstract 64T-441, Notices Amer. Math. Soc. 11 (1964), 679.

4. - Some observations on quasicohesive sets, Michigan Math. J. 11 (1964), $83-87$.

5. C. E. M. Yates, Recursively enumerable sets and retracing functions, Z. Math. Logik Grundlagen Math. 8 (1962), 331-345.

UNIVERSITY OF ILLINOIS 